\title{
ONE-POT-TWO-STEP SYNTHESIS OF BIOLUBRICANT BASE STOCK FROM RUBBER SEED OIL
}

\author{
G.O. Madojemu ${ }^{1}$, E.A. Elimian ${ }^{2}$, M.C. Ejimadu ${ }^{3}$, C.O. Okieimen ${ }^{1}$, and F.E. Okieimen ${ }^{3}$ \\ University of Benin, ${ }^{1}$ Department of Chemical Engineering, Faculty of Engineering \\ ${ }^{2}$ Department of Plant Biology and Biotechnology, Faculty of Life Sciences \\ ${ }^{3}$ Department of Chemistry, Faculty of Physical Sciences, Benin City, Nigeria. \\ *Correspondents Author: madojemugregory@gmail.com, +2348067499126 \\ Received 28 February 2020; accepted 06 April 2020, published online 28 August 2020
}

\begin{abstract}
Biolubricant base stock was synthesized in this work from rubber seed oil in a one-pot-two-step process of epoxidation and hydroxylation. Rubber seed oil was extracted using a Soxhlet apparatus. The in situ epoxidation of the rubber seed oil with peracid (hydrogen peroxide and acetic acid) was analysed and optimized considering three process variables with their range of values given as temperature of $35-50{ }^{\circ} \mathrm{C}$, time of $60-180$ mins and mole ratio of hydrogen peroxide to acetic acid of 1:0.25-1:1 by applying the central composite design of response surface methodology. The ring opening reaction (hydroxylation) of the epoxide to polyhydroxylated oil (lubricant basestock) with ethanol was carried out using the optimum conditions obtained from the epoxidation process. The rubber seed oil, epoxide and lubricant basestock were characterized in terms of physico-chemical properties using standard methods and in terms of functional groups using Fourier Transform Infrared (FTIR) spectroscopy. Maximum epoxide content of $4.85 \%$ and maximum conversion of $71 \%$ of rubber seed oil to epoxide was achieved at a temperature of $50{ }^{\circ} \mathrm{C}$, reaction time of $180 \mathrm{mins}$ and $1: 0.39 \mathrm{~mol} / \mathrm{mol}$ of hydrogen peroxide to acetic acid. The predicted values of the epoxidation process reasonably agreed with the experimental ones and model Rsquared value of about $95 \%$ showed that response surface method can reasonably predict the epoxidation process using a quadratic polynomial model. There was $75 \%$ conversion of the epoxide to polyhydroxylated oil (biolubricant basestock), which represents a very high yield. The formation of epoxides and polyhydroxylated oil lead to modification (improvement) in the properties of rubber seed oil as confirmed by the physico-chemical properties and FTIR spectra analysis of the oil, epoxide and lubricant basestock. The study showed that chemical derivatives of rubber seed oils are an attractive, renewable, and ecofriendly alternative to mineral oils for lubricant formulations.
\end{abstract}

Keywords: Rubber seed oil, epoxidation, hydroxylation, response surface methodology

\subsection{INTRODUCTION}

Petroleum or mineral oil which currently serve as major base stocks for lubricant formulations are not environmental friendly coupled with difficulties for their safe and easy disposal. It is claimed that $50 \%$ of all lubricants sold worldwide end up in the environment via accidental spillage, non-recoverable usage, volatility, industrial and municipal waste, urban runoff, refinery processes and total loss applications (in chainsaw oils, two-stroke engines, concrete mold release oils, exhaust fumes in engines and metal cutting and forming processes) [1,2].

Plant-based oils are showing great potentials nowadays and are highly attractive material to replace the conventional mineral oils for use in lubricant production because they are structurally similar to the long chained hydrocarbons in mineral oils with the characteristics of being renewable, non-toxic, economic and environmentally friendly $[3,4]$. In the past few decades, there have been environmental public awareness and any product that does not meet the environmental standard of biodegradability is considered dangerous to the environment.

Though most currently used lubricants originate from petroleum base stocks, vegetable oils have seen a promising increase in use as biodegradable fluids over the last decade. Environmental concerns as well as economics and performance issues will drive the market share for these oils [5]. Vegetable oils have very low volatility due to the high molecular weight of triglyceride structure and narrow range of viscosity change with temperature. Their ester linkages deliver inherent lubricity and are able to stick to metal surfaces. Further, vegetable oils have more superior solubilizing power for contaminants and additive 
molecules than mineral-based fluids. Some performance limitations of vegetable oil base stocks in lubricant formulations are their poor oxidative stability, deposit-forming tendency, low temperature solidification and low-hydrolytic stability. On the other hand, parameters such as lubricity, antiwear protection, load-carrying capacity, rust prevention, foaming, and demulsibility are mostly additive-dependent [2]. Therefore, when a given vegetable oil is considered as a potential base stock for industrial or automotive application, the base-stock-dependent parameters must be evaluated first. The performance limitations of vegetable oil-based stocks can be overcomed by genetic modification, chemical modification, processing changes, and development in their additive technology [5] such as esterification, epoxidation and hydrogenation [6].

A potentially very attractive plant oil for the synthesis of advanced materials is the oil from rubber seeds of the rubber tree (Hevea brasiliensis). So far, the tree has been cultivated mainly as an industrial crop for the production of natural rubber and valorization of the seeds has received limited attention. Rubber seed oil (RSO) is particularly attractive as it has a relatively high content of unsaturated fatty acids [7, 8], high amount of oil and does not compete with food oils [8].

Response surface methodology (RSM) can be used to evaluate the relative significance of several factors in the presence of complex interactions. RSM answers the question of how to select the levels for the applied factors to obtain the desirable, smallest or largest, value of the response function in a reduced number of experiments [9]. In this work, biolubricant base stock was synthesized by chemically modifying the properties of rubber seed oil via a two-step process of epoxidation and hydroxylation. RSM was employed in the epoxidation of rubber seed oil with peracids to determine the effect of epoxidation process parameters on the epoxide content.

\subsection{MATERIALS AND METHODS}

Already shelled rubber seeds were collected from Rubber Research Institute of Nigeria, Benin City, Nigeria. The kernels were dried, milled and stored for oil extraction and analysis. All reagents used in this work were of analytical grade and were used without further purification. The oil extraction was done following method by Nwabanne and Omoniyi [10]. The extraction process was carried out in a $2000 \mathrm{ml}$
Soxhlet extractor using n-hexane as solvent. n-hexane of $150 \mathrm{ml}$ was poured into a $500 \mathrm{ml}$ round bottom flask and $600 \mathrm{~g}$ of the milled kernels were placed in the thimble and inserted in the center of the extractor. The Soxhlet was heated at $60{ }^{\circ} \mathrm{C}$ (near the boiling point of n-hexane). As the solvent starts boiling, the vapour rose through the vertical tube into the condenser at the top. The liquid condensate dripped into the filter paper thimble in the center which contained the solid sample to be extracted. The extract seeped through the pores of thimble and filled the siphon tube, where it flowed back down into the round bottom flask. This was allowed to continue for $5 \mathrm{~min}$. At the end, the solvent was evaporated using a rotary evaporator and the percentage oil extracted was determined using the expression in Equation (1). The extraction process was repeated severally until the desired amount of the oil needed for further analysis was obtained.

$$
\text { Percentage oil yield }=\frac{\text { mass of oil }}{\text { mass of seed }} \times 100 \%
$$

\subsection{Epoxidation reaction and experimental design}

The epoxidation reaction was carried out in a threenecked round bottom flask ( $250 \mathrm{ml}$ capacity), equipped with a magnetic stirrer, a funnel and a thermometer and the setup was placed in a water bath. The center neck was connected to a reflux condenser and the thermometer was connected to another neck in order to record the reaction temperature that was controlled within $\pm 2{ }^{\circ} \mathrm{C}$. Epoxidation method used was according to Goud et al. [11]. Mass of $25.8 \mathrm{~g}$ of rubber seed oil was placed in the reactor. The calculated amounts of acetic acid and sulphuric acid (catalyst) was added and stirred for 30 mins. Hydrogen peroxide was added to the mixture dropwise at a rate to ensure it was completed in half an hour. The completion of $\mathrm{H}_{2} \mathrm{O}_{2}$ addition was recorded as zero time and the epoxidation reaction was performed according to the experimental design of Table 1 . The mixture in round bottom flask was stirred continuously to avoid zones of high peroxide concentration that could lead to explosive mixtures. After that, the collected samples were centrifuged before determining oxirane oxygen content.

RSM was used to assess the influence of various process parameters to maximize oxirane oxygen content (epoxide content). The experimental design for this reaction was carried out by employing a full factorial rotatable central composite design (RCCD) for three factors with replicates at the centre point and 
star points were used in the investigation by the statistical software package Design-expert ${ }^{\circledR}$ (version 8.0.6; stat-ease, Inc., Minneapolis, USA). The variables used were temperature $\left(x_{1}\right)$, time $\left(x_{2}\right)$, and mole ratio of hydrogen peroxide to acetic acid $\left(x_{3}\right)$, each at low $(-1)$ and high $(+1)$ coded levels. A total of 20 experimental trials that included 8 trials for factorial design, 6 trials for axial points (two for each variable) and 6 trials for replication of the central points were performed. The response, relative conversion (Y) is the average of duplicate determination of oxirane oxygen content. The levels of independent variables were selected based on values obtained in preliminary experiments reported by Okieimen et al. [8]. Table 1 shows the CCD experimental range of values for the process variables considered for the epoxidation process.

Table 1: CCD experimental conditions for the epoxidation process

\begin{tabular}{cccc}
\hline \multirow{2}{*}{ Independent variable } & Symbol & \multicolumn{3}{c}{ Levels } \\
\cline { 3 - 4 } & & 35 & +1 \\
\hline Temperature $\left({ }^{\circ} \mathrm{C}\right)$ & $x_{1}$ & 60 & 60 \\
Time (mins) & $x_{2}$ & $1: 0.25$ & 180 \\
HP:AA (mol/mol) & $x_{3}$ & $1: 1$ \\
\hline
\end{tabular}

$\mathrm{HP}=$ Hydrogen peroxide $\mathrm{AA}=$ Acetic acid

The experimental data were analyzed according to the response surface regression procedure to fit the secondorder polynomial equation as given by Equation (2) in which the level of significance (p-value) of all coefficients was $<0.05$. Based on experimental data shown in Table 1, the regression coefficient was determined to predict the process response as a function of independent variables and their interactions were used to understand the system behaviour.

$$
Y=\beta_{0}+\sum_{i=1}^{n} \beta_{i} x_{i}+\sum_{i=1}^{n} \beta_{i i} x_{i}^{2}+\sum_{i=1}^{n} \sum_{j>1}^{n} \beta_{i j} x_{i} x_{j}
$$

where $Y$ is the response, i.e. the relative conversion to oxirane oxygen (\%), $X_{\mathrm{i}}$ and $X_{\mathrm{j}}$ represent the independent variables, $\beta_{0}$ is constant, $\beta_{\mathrm{i}}$ is linear term coefficient, $\beta_{\mathrm{ii}}$ is the quadratic term coefficient, $\beta_{\mathrm{ij}}$ is cross-term coefficient and ' $n$ ' is the number of process variables studied and optimized during the study. ANOVA was carried out to estimate the effects of process variables and their possible interaction effects on the maximum ethanol yield in the response surface regression procedure. The goodness and best fit of the model was evaluated by a regression coefficient $R^{2}$. The response surface and contour plots were obtained using the fitted quadratic polynomial equation generated from regression analysis by keeping one of the independent variables at central value (0) and varying the other two. A direct Oxirane value indicates the mass of oxygen present in the epoxy groups. Experimentally, oxirane values were determined by the direct method using hydrobromic acid solution in glacial acetic acid [11]. Theoretical oxirane oxygen $\left(\mathrm{OO}_{\text {the }}\right)$ and the relative percentage conversion to oxirane were computed from the following expressions:

$O O_{\text {the }}=\left[\frac{\left(I V_{0} / 2 A_{i}\right)}{100+\left(I V_{0} / 2 A_{i}\right) A_{0}}\right] A_{0} \times 100 \%$

Where $A i$ (126.9) and $A 0$ (16) is the atomic weights of iodine and oxygen respectively, and $I V 0$ is the initial iodine value of the RSO.

Relative conversion $(\%)=\left[\frac{O O_{\text {exp }}}{O O_{\text {the }}}\right] \times 100 \%$

Where $\mathrm{OO}_{\text {exp }}$ is the experimentally determined content of oxirane oxygen in $100 \mathrm{~g}$ of oil, and $\mathrm{OO}_{\text {the }}$ is the theoretically maximum oxirane oxygen content in 100 $\mathrm{g}$ of oil [12]. Each oxirane oxygen content analysis was duplicated, and the average values were reported.

\subsection{Hydroxylation of the epoxide (Ring opening reaction)}

The ring-opening reaction was carried out in similar set up as the epoxidation process. This hydroxylation reaction of the epoxide to open its ring was performed following optimal conditions reported by Tucor et al. [13] with modification in the type of catalyst used and reaction time. For the hydroxylation step, $2 \mathrm{wt} \%$ of $\mathrm{H}_{2} \mathrm{SO}_{4}$ (catalyst) was used in the ring-opening reaction for $4 \mathrm{hr}$ at a temperature of $80^{\circ} \mathrm{C}$ using $10: 1$ mole of 
ethanol to epoxidised rubber seed oil (ERSO). The ethanol and catalyst were mixed and heated to $80^{\circ} \mathrm{C}$, then ERSO was added to start the reaction process. Samples were withdrawn after the reaction time, quenched and centrifuged to separate the solid catalyst. The residual solvent was then evaporated by heating at $80^{\circ} \mathrm{C}$ under high vacuum. The product of the hydroxylation process is known as polyhydroxylated oil or hydroxylated rubber seed oil (HRSO).

The oxirane conversion to HRSO was calculated using Equation (5) as:

Oxirane conversion $=\frac{\left(O N_{i}-O N_{f}\right)}{O N_{i}}$

where $\mathrm{ON}_{\mathrm{i}}$ and $\mathrm{ON}_{\mathrm{f}}$ are the initial and final oxirane numbers, respectively.

\subsection{Characterization of RSO, ERSO and HRSO}

RSO, ERSO and HRSO were characterized in terms of physico-chemical properties using standard methods and in terms of functional groups using FTIR analysis using a Perkin Elmer Spectrum (ASC III PEDS 1.60). The samples were used in the form of potassium bromide (KBR) discs which were prepared by grinding $1 \mathrm{mg}$ sample/100 mg pre-dried KBR. The spectra were recorded in the range of $4400-350 \mathrm{~cm}^{-1}$.

\subsection{RESULTS AND DISCUSSION}

\subsection{Analysis of epoxide yield}

Oil yield of $43.68 \%$ was obtained after extraction with $150 \mathrm{ml}$ of $99.5 \% \mathrm{n}$-hexane. This amount of oil was extracted from $600 \mathrm{~g}$ of the rubber seed kernel using Soxhlet apparatus. This amount represents a high yield of oil and is in accordance with the range of $35-45 \%$ RSO yield reported by Nwokolo et al. [14]. The iodine value of the RSO was found to be 117.0 g. $\mathrm{I}_{2} / 100 \mathrm{~g}$ and the theoretical yield of oxirane oxygen was calculated to be $6.869 \%$ using Equation (3). The epoxide content was determined from $0.05 \mathrm{~mol}$ of RSO and $1.00 \mathrm{~mol}$ $(34.01 \mathrm{~g})$ of hydrogen peroxide which was fixed all epoxidation experimental runs varying only the number of moles of acetic acid. Table 2 gives the experimental matrix and epoxide content obtained after epoxidation of RSO with per acid. The maximum observed relative conversion of RSO to epoxide content was $70.90 \%$ while the lowest was $6.48 \%$. The maximum relative conversion was obtained at a temperature of $50^{\circ} \mathrm{C}$, time of $180 \mathrm{mins}$ and $0.25 \mathrm{~mol}$ of acetic acid (AA). The predicted vs. actual plot for the relative conversion of RSO to epoxide is given by Figure 1 which showed a strong linear correlation between the predicted and the actual values, which indicate model is satisfactory. Table 3 gives the analysis of variance (ANOVA) of the relative conversion of RSO to epoxide. P-values less than 0.05 indicate model terms/factors are significant i.e. change in the factor leads to a corresponding change in relative conversion of RSO to oxirane oxygen at a significant level of 5\%. From Table 3, high F-value of 32.32 and very low $p$-value of $<0.0001(<0.05)$ indicate model is highly significant. Among the main/linear factors, temperature $\left(x_{1}\right)$ and time $\left(x_{2}\right)$ are highly significant while variation in the molar ratio of hydrogen peroxide to acetic acid $\left(x_{3}\right)$ is insignificant on the relative conversion of RSO to ERSO. Only the interaction of time and mole of acetic acid $\left(x_{2} x_{3}\right)$ were found to be significant among the interaction factors, while all quadratic factors $\left(x_{1}^{2}, x_{2}^{2}\right.$, and $\left.x_{3}^{2}\right)$ were significant model terms. There is insignificant lack of fit with $\mathrm{p}$ value of 0.0670 which shows the quadratic model reasonably predicted the epoxidation process. Equation (6) gives the empirical model of the epoxidation process, showing the relationship between relative conversion of RSO to ERSO and the variables considered (significant only) in coded values.

$Y(\%)=63.09+7.40 x_{1}+7.57 x_{2}+0.58 x_{3}-$ $18.27 x_{2} x_{3}-4.81 x_{1}^{2}-6.62-17.45 x_{3}^{2}$

In a regression equation, when an independent variable has a positive sign, it means that an increase in the variable will cause an increase in the response while a negative sign will result in a decrease in the response $[15,16]$ Hence, an increase in temperature $\left(x_{1}\right)$, time $\left(x_{2}\right)$ and molar ratio of hydrogen peroxide to acetic acid $\left(x_{3}\right)$ will lead to an increase in the relative conversion of RSO to OO. Epoxidation time $\left(x_{2}\right)$ showed the highest significant effect on the relative conversion to ERSO while mole of acetic acid showed the least effect among the main/linear variables. The coefficient of regression $\mathrm{R}^{2}$ was used to validate the fitness of the model equation. Table 4 gives an $\mathrm{R}^{2}$ of a high value of 0.9496 , showing that $94.96 \%$ of the variability in the relative conversion of the process can be explained by the model. There is also positive significant correlation between the adjusted R-squared value (0.9131) and the predicted R-squared value (0.6477), which shows that the model reasonably 
predicted the epoxidation process using a quadratic polynomial.

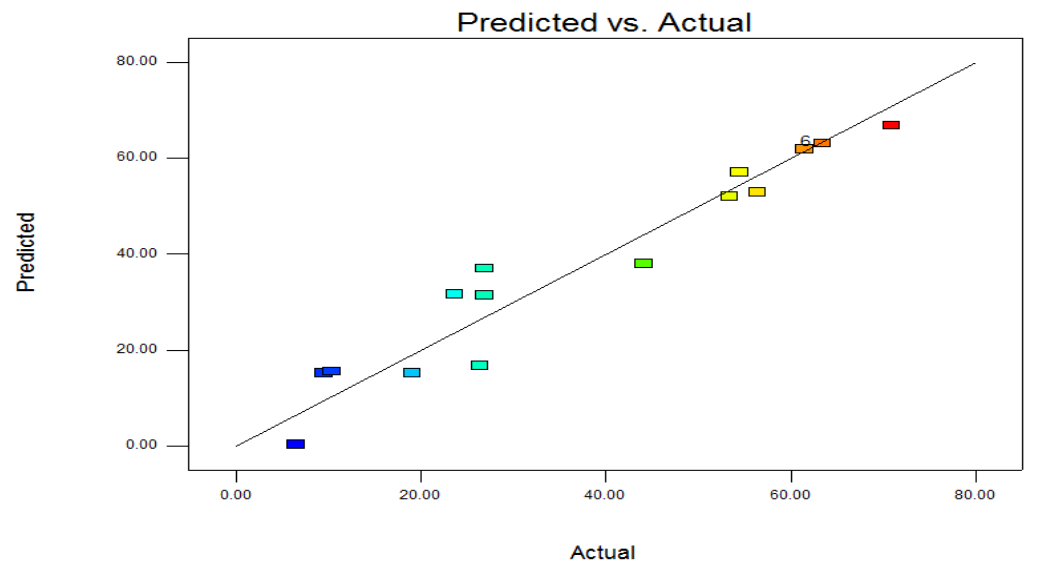

Figure 1: Predicted vs. actual plot for the relative conversion of RSO to epoxide

Table 2: Experimental design matrix with relative conversion to oxirane oxygen

\begin{tabular}{ccccccccc}
\hline \multirow{2}{*}{$\begin{array}{c}\text { Std } \\
\text { order }\end{array}$} & \multicolumn{3}{c}{ Coded factors } & \multicolumn{3}{c}{ Actual factors } & \multicolumn{2}{c}{ Y (\%) } \\
\cline { 2 - 8 } & $\boldsymbol{x}_{\mathbf{1}}$ & $\boldsymbol{x}_{\mathbf{2}}$ & $\boldsymbol{x}_{\mathbf{3}}$ & $\boldsymbol{x}_{\mathbf{1}}=\boldsymbol{t e m p} \boldsymbol{~}$ & $\boldsymbol{x}_{\mathbf{2}}=\boldsymbol{t i m e}$ & $\boldsymbol{x}_{\mathbf{3}}=\boldsymbol{m o l}(\boldsymbol{A A})$ & Actual & Predicted \\
\hline 1 & -1 & -1 & -1 & 35 & 60 & 0.25 & 6.48 & 0.40 \\
2 & 1 & -1 & -1 & 50 & 60 & 0.25 & 19.01 & 15.19 \\
3 & -1 & 1 & -1 & 35 & 180 & 0.25 & 53.42 & 52.06 \\
4 & 1 & 1 & -1 & 50 & 180 & 0.25 & 70.90 & 66.86 \\
5 & -1 & -1 & 1 & 35 & 60 & 1.00 & 44.12 & 38.09 \\
6 & 1 & -1 & 1 & 50 & 60 & 1.00 & 56.44 & 52.88 \\
7 & -1 & 1 & 1 & 35 & 180 & 1.00 & 26.39 & 16.69 \\
8 & 1 & 1 & 1 & 50 & 180 & 1.00 & 26.87 & 31.49 \\
9 & -1.68 & 0 & 0 & 29.89 & 120 & 0.625 & 26.88 & 37.05 \\
10 & 1.68 & 0 & 0 & 55.11 & 120 & 0.625 & 61.5 & 61.93 \\
11 & 0 & -1.68 & 0 & 42.5 & 19.09 & 0.625 & 23.66 & 31.64 \\
12 & 0 & 1.68 & 0 & 42.5 & 220.91 & 0.625 & 54.47 & 57.09 \\
13 & 0 & 0 & -1.68 & 42.5 & 120 & 0.01 & 9.48 & 15.20 \\
14 & 0 & 0 & 1.68 & 42.5 & 120 & 1.25 & 10.32 & 15.57 \\
15 & 0 & 0 & 0 & 42.5 & 120 & 0.625 & 63.16 & 63.09 \\
16 & 0 & 0 & 0 & 42.5 & 120 & 0.625 & 63.43 & 63.09 \\
17 & 0 & 0 & 0 & 42.5 & 120 & 0.625 & 63.06 & 63.09 \\
18 & 0 & 0 & 0 & 42.5 & 120 & 0.625 & 63.23 & 63.09 \\
19 & 0 & 0 & 0 & 42.5 & 120 & 0.625 & 63.41 & 63.09 \\
20 & 0 & 0 & 0 & 42.5 & 120 & 0.625 & 63.36 & 63.09 \\
\hline
\end{tabular}


Table 3: ANOVA of the relative conversion of RSO to epoxide

\begin{tabular}{cccccc}
\hline Source & $\begin{array}{c}\text { Sum of } \\
\text { squares }\end{array}$ & Df & $\begin{array}{c}\text { Mean } \\
\text { square }\end{array}$ & $\begin{array}{c}\text { F } \\
\text { Value }\end{array}$ & $\begin{array}{c}\text { p-value } \\
\text { Prob > F }\end{array}$ \\
\hline Model & 8850.0302 & 7 & 1264.2900 & 32.3236 & $<0.0001^{*}$ \\
$x_{1}$-temperature & 747.4490 & 1 & 747.4490 & 19.1097 & $0.0009^{*}$ \\
$x_{2}$-time & 782.0544 & 1 & 782.0544 & 19.9945 & $0.0008^{*}$ \\
$x_{3}$-no. of moles (AA) & 4.5136 & 1 & 4.5136 & 0.1154 & 0.7400 \\
$x_{2} x_{3}$ & 2669.2471 & 1 & 2669.2471 & 68.2436 & $<0.0001^{*}$ \\
$x_{1}^{2}$ & 333.9367 & 1 & 333.9367 & 8.5376 & $0.0128^{*}$ \\
$x_{2}^{2}$ & 632.8917 & 1 & 632.8917 & 16.1809 & $0.0017^{*}$ \\
$x_{3}^{2}$ & 4163.1043 & 1 & 4163.1043 & 106.4364 & $<0.0001^{*}$ \\
Residual & 469.3625 & 12 & 39.1135 & & \\
Lack of fit & 469.3625 & 7 & 67.0518 & 6.2289 & 0.0670 \\
Pure error & 53.823 & 5 & 10.7646 & & \\
Cor. Total & 9319.3927 & 19 & & & \\
\hline \multicolumn{7}{c}{$=$ significant }
\end{tabular}

Table 4: Model summary statistics

\begin{tabular}{ccccccc}
\hline Source & $\begin{array}{c}\text { Std. } \\
\text { Dev. }\end{array}$ & R-Squared & $\begin{array}{c}\text { Adjusted } \\
\text { R-Squared }\end{array}$ & $\begin{array}{c}\text { Predicted } \\
\text { R-Squared }\end{array}$ & PRESS & \\
\hline Linear & 22.0630 & 0.1643 & 0.0076 & -0.3518 & 12597.530 & \\
2FI & 19.7605 & 0.4553 & 0.2039 & -0.0190 & 9496.784 & \\
Quadratic & 6.5300 & 0.9496 & 0.9131 & 0.6477 & 3283.118 & Suggested \\
Cubic & 0 & 1 & 1 & & + & Aliased \\
\hline
\end{tabular}

Figure 2 (a) gives the response surface plot showing the interaction effect of epoxidation time and mole acetic acid on the relative conversion of RSO to epoxide while Figure 2 (b) gives the corresponding contour plot.

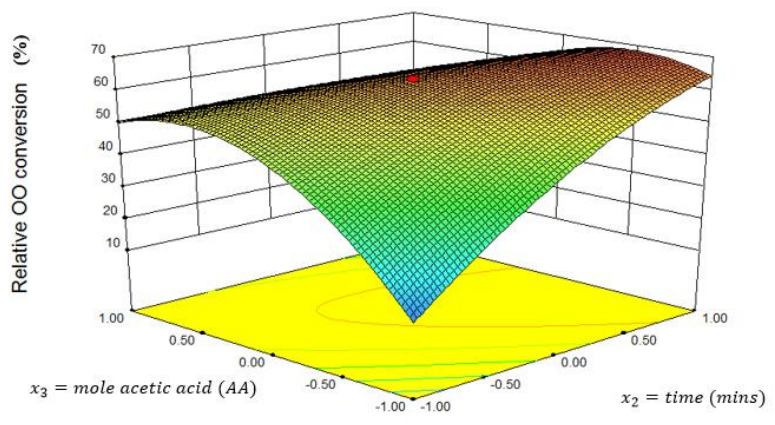

(a)

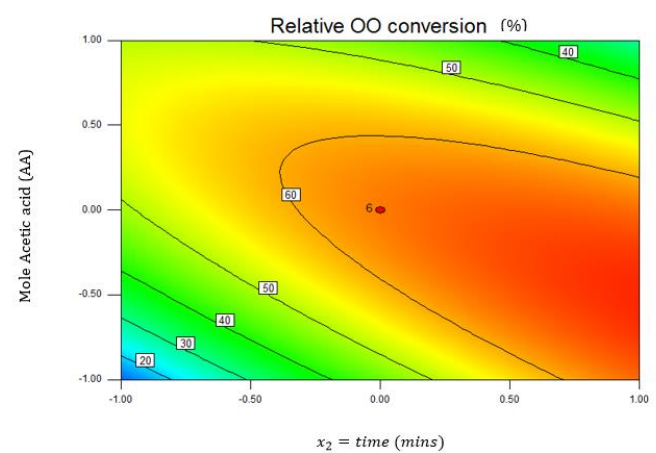

(b)

Figure 2.(a) Response surface plot (b) corresponding contour plot showing the interaction effect of epoxidation time and mole of acetic acid (AA). 
The relationship between independent and dependent variables is illustrated in three dimensional representation of the response surfaces as by Figure 2 (a) and two-dimensional contour plots, Figure 2 (b) generated by the model gives the effect of interaction of time (60-180 mins) and $\mathrm{mol} / \mathrm{mol}$ hydrogen peroxide to acetic acid (0.25-1.00) on the relative conversion of RSO to epoxide at constant temperature. As both epoxidation time and mole of AA increased the relative conversion of RSO to $\mathrm{OO}$ also increased. The achieved maximum desirability of 0.984 means that it is possible to reach maximum relative conversion target. The maximum oxirane oxygen of $4.85 \%$ and maximum relative conversion of the epoxidation process of $70.90 \%$ was obtained at a temperature of $50^{\circ} \mathrm{C}$, time of $180 \mathrm{mins}$ and $0.39 \mathrm{~mol} / \mathrm{mol}$ hydrogen peroxide to acetic acid.

\subsection{Physico-chemical properties}

The ring opening reaction (hydroxylation) of the epoxide gave a polyhydroxylated oil with the following physico-chemical properties. The esterification process gave the desired biolubricant basestock (ester derivative) with $75 \%$ conversion of epoxide to HRSO (biolubricant base stock). Table 5 gives the physicochemical properties of RSO, ERSO and HRSO.

\section{Table 5: Physico-chemical properties of HRSO, ERSO and RSO}

\begin{tabular}{lccc}
\hline \multicolumn{1}{c}{ Properties } & RSO & ERSO & HRSO \\
\hline Colour & Yellow & Yellow & Yellow \\
Acid value $\left(\mathrm{mgKOH} \cdot \mathrm{g}^{-1}\right)$ & 39.97 & 34.70 & 32.50 \\
Saponification value $(\mathrm{mg} \mathrm{KOH} / \mathrm{g})$ & 204.84 & 215.86 & 232.12 \\
Specific gravity @ $30^{\circ} \mathrm{C}$ & 0.912 & 1.031 & 1.001 \\
Viscosity @ $30^{\circ} \mathrm{C}(\mathrm{cp})$ & 60.90 & 161.50 & 166.57 \\
Refractive index () & 1.4709 & 1.4690 & 1.4405 \\
Iodine value $\left(\mathrm{g} \mathrm{I}_{2} \cdot\left[100 \mathrm{~g}^{-1}\right)\right.$ & 117.00 & 17.12 & 9.88 \\
Epoxide content $(\%)$ & - & 4.85 & 1.41 \\
\hline
\end{tabular}

Figure 3 (a), (b) and (c) gives the FTIR spectra of RSO, ERSO and HRSO respectively. There are similarities in the FTIR spectra of HRSO, ERSO and RSO as depicted by the Figures. The difference in the spectra for RSO and ERSO is that ERSO shows absorption at $864.33 \mathrm{~cm}^{-1}$. Okieimen et al. [17] reported this difference by absorption at $824 \mathrm{~cm}^{-1}$ which denote the presence of epoxide while Gamage et al. [18] reported the presence of epoxide after epoxidation of waste cooking oil at $838.00 \mathrm{~cm}^{-1}$. Farias et al. [19] identified that via oxirane ring opening reaction, $\mathrm{OH}$ functional groups can be derived from epoxy functional groups. In this study, FTIR spectra exhibited no trace of $\mathrm{OH}$ absorption peak at approximately $3000-3300 \mathrm{~cm}^{-1}$ as shown by the spectra in Figure 3 (b) which represents no oxirane cleavage during the epoxidation reaction. This range of no oxirane clavage was reported to be 3000 to 3500 $\mathrm{cm}^{-1}$ for epoxidized waste cooking oil as reported by Gamage et al. [18]. The presence of $\mathrm{OH}$ at $3369.0 \mathrm{~cm}^{-1}$ showed oxirane cleavage. This must have been due to epoxy ring opening to form hydroxylated product which resulted in a sharp and strong $\mathrm{O}-\mathrm{H}$ stretching node at about $3418.00 \mathrm{~cm}^{-1}$ in the IR spectrum of hydroxylated oil shown in Figure 3(c). 
J. Chem. Soc. Nigeria, Vol. 45, No.5, pp 935 - 944 [2020]

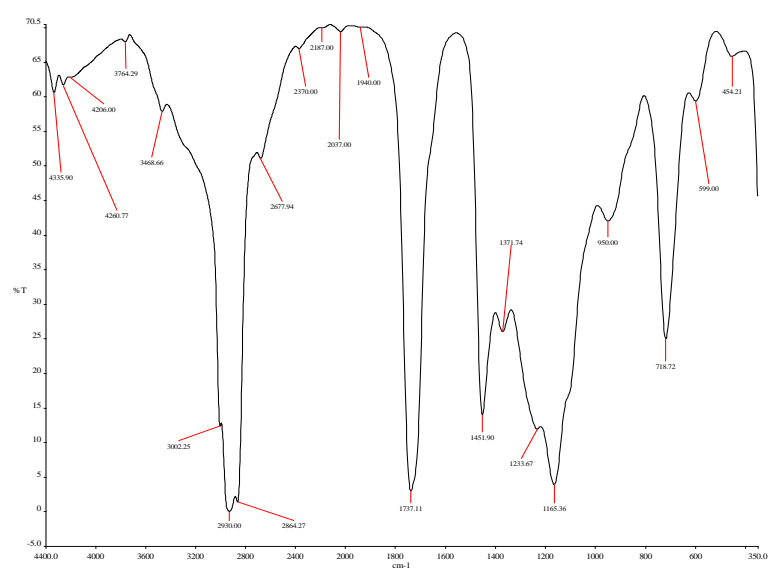

(a)

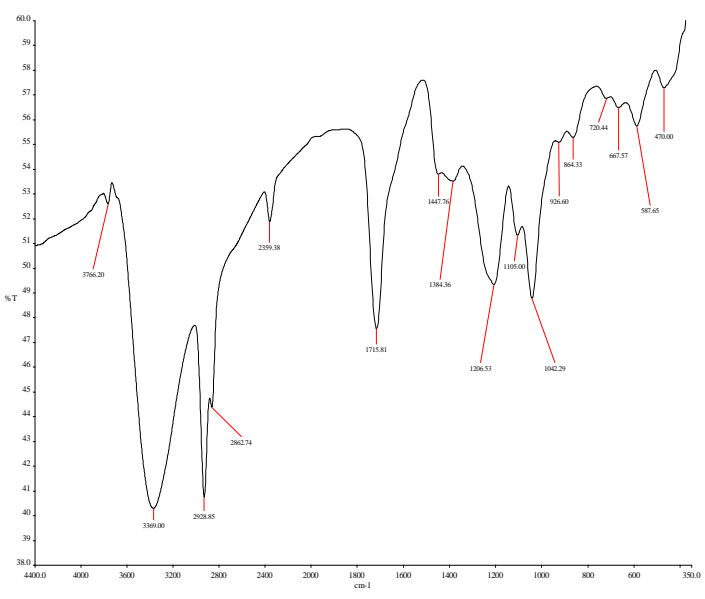

(b)

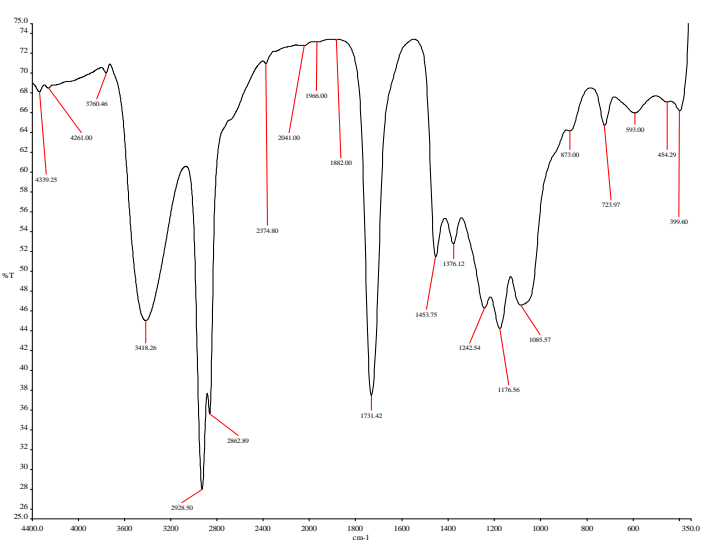

(c)

Figure 3. FTIR spectra of (a) RSO (b) ERSO and (c) HRSO 


\subsection{CONCLUSION}

In this work, biolubricant basestock was synthesized from rubber seed oil by a one-pot-two-step process of epoxidation and hydroxylation. The epoxidation process was designed by varying temperature, time and mole ratio of hydrogen peroxide to acetic acid and the relative conversion of rubber seed oil to epoxide was designed, analyzed and optimized employing the central composite design of response surface methodology. Soxhlet apparatus gave $43.6 \%$ of rubber seed oil which represents a very high yield of oil from the kernel. Maximum epoxide content of $4.85 \%$ and maximum relative conversion of $71 \%$ to epoxide of the rubber seed oil was achieved at a temperature of $50{ }^{\circ} \mathrm{C}$, reaction time of $3 \mathrm{hrs}$ and $0.39 \mathrm{~mol}$ of acetic acid. There was $75 \%$ conversion of the epoxide to polyhydroxylated oil (biolubricant basestock), which represent a very high yield. The predicted values correlated reasonably with experimental values and high R-squared value of over $96 \%$ showed that response surface method can reasonably predict the process using a quadratic polynomial model. The formation of epoxides and polyhydroxylated oil lead to modification (improvement) in the properties of rubber seed oil as confirmed by the physico-chemical properties and FTIR spectra analysis of the oil, epoxide and polyhydroxylated oil. This study shows that chemical derivatives of rubber seed oil are an attractive, renewable, and ecofriendly alternative for mineral oils for the synthesis of lubricant formulations.

\section{REFERENCES}

[1] I. Gawrilow (2004), Vegetable oil usage in lubricants, INFORM 15(11): 702-705.

[2] S. Z. Erhan, B. K. Sharma, Z. Liu and A. Adhvaryu (2008), Lubricant base stock potential of chemically modified vegetable oils, Journal of Agricultural and Food Chemistry, 56: 8919-8925.

[3] N. J. Fox, and G. W. Stachowiak. (2007), Vegetable oil-based lubricants-A review of oxidation, Tribology International, 40(7): 1035-1046.

[4] P. Nagendramma, and S. Kaul (2012), Development of ecofriendly/biodegradable lubricants: An overview, Renewable and Sustainable Energy Reviews, 16:764-774.
[5] A. Adhvaryu, S. Z. Erhan (2002), Epoxidized soybean oil as a potential source of high temperature lubricants, Ind. Crop. Prod., 15(3): 247-254.

[6] S. Z. Erhan, B. K. Sharma and J. M. Perez (2006), Oxidation and low temperature stability of vegetable oil-based lubricants, Ind. Crop. Prod., 24:292-299.

[7] F. E. Okieimen (2002), Studies in the utilisation of Epoxidized vegetable oils as thermal stabiliser for polyvinyl chloride, Ind. Crops. Prod., 15: 71-75.

[8] F. E. Okieimen, O. I. Bakare and C. O. Okieimen (2002), Studies on the epoxidation of rubber seed oil, Ind. Crop. Prod., 15(2): 139-144.

[9] G. Hanrahan and K. Lu (2006), Application of factorial designs and response surface methodology in modern experimental design and optimization, Rev Anal Chem., 36:141-151.

[10] J. T. Nwabanne and M. H. Omoniyi (2010), The effect of process variables on the physico-chemical properties and oil yield of rubber seed, Journal of Engineering and Applied Sciences, 6: 47-51.

[11] V. V. Goud, S. Dinda, A. V. Patwardhan and N. C. Pradhan (2008), Epoxidation of Cottonseed Oil by aqueous hydrogen peroxide catalyzed by Liquid inorganic acids, Bioresour. Technol., 99(9): 37373744.

[12] V.B. Borugadda, and V. Goud (2014), Synthesis of Waste Cooking Oil Epoxide as a Bio-lubricant Base Stock: Characterization and optimization study, $J$. Bioprocess Eng. Biorefinery, 3: 1-14.

[13] R. Tucor, R. Tesser, R. D. Vitiello, V. Russo, S. Andini, and M. Di Serio (2017), Synthesis of biolubricant Basestocks from epoxidised soybean oil, Catalysts, 7(309): 1-11.

[14] E. Nwokolo, D. D. Kitts and J. Kanhai (1988), Serum and liver lipids of rats fed rubber seed oil, Plant Foods for Human Nutrition, 38: 145-153.

[15] V. L. Russell (2009), Response surface methods in R, using RSM, J. Stat. Ease Software, Mineapolis, USA.

[16] O. Ocholi, M. Menkiti, M. Auta and I. Ezemagu, (2018), Optimization of the operating parameters for 
the extractive synthesis of biolubricant from sesame seed oil via response surface methodology, Egyptian Journal of Petroleum, 27: 265-275.

[17] F. E. Okieimen, C. Pavithran and I. O. Bakare (2005), Epoxidation and hydroxylation of rubber seed oil: one - pot multi-step reactions, European Journal of Lipid Science and Technology, 107(5): 330-336.
[18] P. K. Gamage, M. O'Brien and L. Karunanayake (2009), Epoxidation of some vegetable oils and their hydrolysed products with peroxyformic acid-optimised to industrial scale, J. Natl. Sci. Found Sri Lanka, 37: 229-240.

[19] M. Farias, M. Martnelli, and D. P. Bottega (2010), Epoxidation of soybean oil using a homogeneous catalytic system based on a molybdenum (VI) complex, Applied Catalysis A General, 384(1):213-2 
J. Chem. Soc. Nigeria, Vol. 45, No.5, pp 935 - 944 [2020] 